\title{
Bronchoalveolar Lavage Surfactant Protein A, B, and D Concentrations in Preterm Infants Ventilated for Respiratory Distress Syndrome Receiving Natural and Synthetic Surfactants
}

\author{
MICHAEL W. BERESFORD AND NIGEL J. SHAW \\ Neonatal Unit, Liverpool Women's Hospital, Liverpool L8 7SS, United Kingdom.
}

\begin{abstract}
ABS
Surfactant proteins (SPs) play an important role in surfactant
metabolism and function. Understanding their relative contribu-
tion to clinical outcome remains incomplete. Exogenous surfac-
tants differ in their SP content and physiologic effects. The aims
of this study were to measure bronchoalveolar lavage (BAL) SP
concentrations from preterm infants ventilated for respiratory
distress syndrome and to assess their association with clinical
outcome. Fifty preterm infants randomized to receive a natural or
synthetic surfactant were lavaged each day for the first week and
twice weekly thereafter using a standardized nonbronchoscopic
technique. BAL SP-A, SP-B, and SP-D concentrations were
measured using ELISA. Median BAL SP-A, SP-B, and SP-D
concentrations for the whole cohort rose significantly during the
first postnatal week ( $p<0.05$ ). SP-A concentration did not differ
between outcome groups. BAL SP-B concentration rose signif-
icantly in lungs that were not supplemented with SP-B. Infants
dying had significantly lower BAL SP-B concentrations on d 2
\end{abstract}
Pulmonary surfactant is a complex, surface-active substance fundamental to lung physiology in health and disease (1). Although formed primarily of lipids, elucidation of the role of SP-A, SP-B, SP-C, and SP-D remains incomplete (1). SP-A and SP-D are hydrophilic proteins belonging to the collectin family of proteins $(1,2)$. SP-A is the predominant surfactant protein in size and quantity (3). The locus of the SP-A genes is on the long arm of chromosome 10 and is associated with that of the SP-D gene (4). SP-A and SP-D are large, complex glycoproteins with monomeric structures (2). Monomers form trimers that cluster to form a bouquetlike octadecamer structure for SP-A and a quaternary cruciate structure for SP-D. Human SP-B is a single gene product of chromosome 2 (4). It is a small protein, rich in hydrophobic and cationic amino acids (1).

Received November 19, 2001; accepted August 26, 2002.

Correspondence: Michael W. Beresford, M.D., Neonatal Unit, Liverpool Women's Hospital, Crown Street, Liverpool L8 7SS, U.K.; e-mail: m_beresford@yahoo.com

Supported by The Newborn Appeal, Serono Laboratories, and Britannia Pharmaceuticals.

DOI: 10.1203/01.PDR.0000054653.89527.F8 and 6 compared with survivors. BAL SP-D concentrations were significantly lower on $\mathrm{d} 2$ and 3 among infants in supplemental oxygen on d 28 compared with those in air. BAL SP-A and SP-D concentrations did not differ significantly between infants randomized to receive a natural or synthetic surfactant. Lower BAL SP-B and SP-D but not SP-A concentrations were associated with worse clinical prognosis. (Pediatr Res 53: 663-670, 2003)
BAL, bronchoalveolar lavage
Abbreviations
CLD, chronic lung disease of prematurity
IUGR, intrauterine growth retardation
IVH, intraventricular hemorrhage
IQR, interquartile range
NB-BAL, nonbronchoscopic BAL
RDS, respiratory distress syndrome
SP, surfactant protein

SP-A and SP-B play important roles in surfactant storage and in tubular myelin and surfactant monolayer formation (1). Acting in synergy, they reduce surface tension and improve lung compliance (5). SP-A regulates alveolar surfactant concentration through inhibition of surfactant exocytosis and enhanced reuptake of surfactant into cells (6). SP-A reduces surfactant inhibition by plasma proteins $(7,8)$. SP-A and SP-D have a range of actions within the innate immune system, including enhancement of leukocyte chemotaxis and function and promoting cytokine release $(2,9)$. SP-D knockout mice have multiple abnormalities of surfactant form and metabolism, indicating a role in surfactant homeostasis (10). However, the complete range of actions of SP-D is still unknown (2).

RDS is the commonest preterm neonatal respiratory disorder resulting in significant morbidity and mortality. Specific SP-A genotypes are associated with increased incidence of RDS (11). Congenital deficiency of SP-B and mutations in the SP-B gene are associated with refractory neonatal respiratory failure (12). Infants dying of RDS appear to show a failure in the normal postnatal rise in lavage SP-A concentrations (13), and 
a low SP-A to phospholipid ratio is associated with poor outcome (7). However, all existing reports of lavage SP-A concentrations in preterm infants with RDS $(7,13-21)$ have used a technique of tracheal aspiration known to sample proximal airways within the bronchial tree (22). In addition, several of these studies $(14-16,20,21)$ standardized for dilutional effects of lavage using unconventional markers of dilution (23).

Surfactant therapy significantly improves survival from RDS. Two studies report SP concentrations in preterm placebo-controlled surfactant studies $(7,16)$. No published data evaluate concentrations in randomly assigned comparisons of surfactant preparations. Exogenous surfactants differ in properties and clinical efficacy. Natural surfactants including poractant alfa (Curosurf, Serono, Laboratories (UK) Ltd, Welwyn Garden City, UK) contain SP-B and SP-C, whereas synthetic surfactants such as pumactant (Artificial Lung Expanding Compound, Britannia Pharmaceuticals, Redhill, UK) contain no SPs. No commercial surfactant currently available contains either SP-A or SP-D. Both poractant alfa (24) and pumactant (25) have a significant impact on outcome from RDS and, until recently, were the most widely used surfactants in the United Kingdom (26). There are no data investigating the effects of pumactant, and minimal data exploring the effects of poractant alfa (21), on BAL SP concentration. Few studies have explored postnatal changes in lavage SP-B in preterm infants with RDS $(14,16)$. There are no data exploring the clinical consequences of SP-D deficiency in preterm infants. Despite animal models suggesting a contribution of SP deficiency to the pathophysiology of CLD (27), there is a paucity of data exploring their concentrations in human infants with CLD.

The aims of this study were to measure BAL SP-A, SP-B, and SP-D concentrations from preterm infants ventilated for RDS and randomized to receive a natural or synthetic surfactant, and to assess their associations with clinical outcome.

\section{METHODS}

Infants were recruited to this study between May 1998 and October 1999 at the Liverpool Women's Hospital, United Kingdom.

Subjects. All infants participated in a randomized controlled trial comparing pumactant with poractant alfa surfactants (26) and received at least one dose of exogenous surfactant. Infants randomized consecutively to the trial in Liverpool were studied. Infants were eligible for study if they were born with a gestational age less than $30 \mathrm{wk}$ with presumed surfactant deficiency. Infants were deemed surfactant deficient if, in the opinion of the attending clinician, they had clinical signs of respiratory distress and required ventilation. Infants were excluded if they had a congenital malformation likely to affect mortality or respiratory outcome. No infant received corticosteroids after birth during the first postnatal week. All infants were ventilated using conventional mandatory ventilation according to established unit guidelines.

The local pediatric research ethics committee approved the study, and written informed parental consent was obtained before patient entry. Demographic and outcome data were collected prospectively. Reason for delivery of infants by cesarean section was defined as the primary obstetric diagnosis appearing in the maternal notes warranting intervention. These included suspected chorioamnionitis, diagnosed on the basis of maternal pyrexia, rising markers of infection, and uterine tenderness; IUGR, defined as fetal growth 2 SD below the mean for gestational age; and fetal distress, diagnosed on the basis of cardiotocographic and fetal blood gas monitoring. Clinical outcomes included CLD, defined as dependency on supplemental oxygen at 28 postnatal days or at 36 postmenstrual weeks; pneumothorax, defined as intrathoracic, extrapulmonary air leak requiring insertion of chest drain; pulmonary hemorrhage, defined as spontaneous appearance of blood or blood-stained fluid in the endotracheal tube; and severe IVH, defined as IVH with ventricular enlargement (28) or parenchymal hemorrhagic lesions (29).

Protocol for BAL and sample processing. BAL took place at the time of routine suctioning using a standardized distal wedge-catheter NB-BAL technique (23). Infants were positioned supine with their heads turned toward the left. Cardiorespiratory monitoring continued throughout. Inspired oxygen concentration was increased just before performing BAL to maintain oxygen saturations $>90 \%$ throughout the procedure. Straight, snub-nosed, end-hole suction catheters were used with mucus suction traps. Suction was provided using portable resuscitaires with variable suction pressure control (Airshields Resuscitaire, Hatboro, U.K.). Each lavage sequence used sterile equipment. After disconnecting the endotracheal tube from the ventilator, an F6 suction catheter was carefully inserted until slight resistance was felt. A $1.0 \mathrm{~mL} / \mathrm{kg}$ (up to a maximum volume of $1.0 \mathrm{~mL}$ ) aliquot of normal saline was instilled gently via the suction catheter, followed by a $0.5-\mathrm{mL}$ air bolus to clear the catheter dead space. The catheter was then connected to a suction trap to which $80-100 \mathrm{~mm} \mathrm{Hg}$ of suction pressure was applied while simultaneously withdrawing the catheter from the endotracheal tube. The infant was reconnected to the ventilator and monitored to ensure a stable state. The whole process was then repeated using a second aliquot of normal saline. The two aliquots were pooled and centrifuged at 2000 rpm for 5 min (Heraeus Sepatech Biofuge-15 centrifuge, Germany). Sample volumes were quantified to the nearest $10 \mu \mathrm{L}$ by pipetting. Cell-free supernatant was aliquoted into polypropylene microtubes and frozen at $-70^{\circ} \mathrm{C}$ within $15 \mathrm{~min}$ of collection. Samples were processed within 12 mo of collection and thawed once, immediately before analysis. Day 1 samples were collected within the first $24 \mathrm{~h}$ after delivery, at least $6 \mathrm{~h}$ after the last surfactant administration. Samples were collected on a daily basis for the first week and twice a week thereafter until extubation.

Surfactant protein ELISAs. SP-A and SP-B were quantified using antigen-capture sandwich ELISA developed and validated at the Children's Hospital Medical Center, Cincinnati, $\mathrm{OH}$, U.S.A., and used in published studies of BAL surfactant (30). Standards and antibodies (unless otherwise stated) were a gift from Professor J.A. Whitsett (Children's Hospital Medical Center, Cincinnati, OH, U.S.A.) and Dr. M. Kerr (University of Glasgow, Scotland, U.K.). The ELISA used to quantify SP-D has been used in published studies of BAL surfactant (31), and antibodies and standards were kindly donated by Dr. H. Clark 
and Dr. P. Strong, Immunochemistry Unit, Medical Research Council, Oxford, U.K. An outline of the ELISA methodology for each SP is presented in brief.

For SP-A analysis, a 1:500 dilution of goat anti-human SP-A antibody in $0.1 \mathrm{M}$ sodium bicarbonate was incubated overnight at $4^{\circ} \mathrm{C}$. "Ohio buffer" was prepared as $5 \%$ BSA in $0.15 \mathrm{M}$ sodium chloride and $0.01 \mathrm{M}$ Tris ( $\mathrm{pH}$ 7.4). SP-A blocking buffer (2.5\% human albumin, $2.5 \%$ goat serum in Ohio buffer) was added to each well $(300 \mu \mathrm{L})$ and left for $90 \mathrm{~min}$ at room temperature. Stock SP-A standards were diluted 1:4 in $0.5 \%$ nonionic detergent NP-40 in PBS (PBS-NP40) to give a working concentration of $250 \mathrm{ng} / \mathrm{mL}$. Serial dilutions using PBSNP40 of standard SP-A and lavage samples diluted 1:3200 and 1:6400 while on ice were incubated for $2 \mathrm{~h}$ along with a blank (PBS-NP40). Polyclonal rabbit anti-human SP-A antibody was diluted 1:400 in SP-A blocking buffer and incubated for $1 \mathrm{~h}$.

For SP-B analysis, wells were coated overnight with $0.1 \mathrm{M}$ sodium bicarbonate at $4^{\circ} \mathrm{C}$. SP-B blocking buffer $(0.5 \%$ human albumin in Ohio buffer) was added to each well $(300 \mu \mathrm{L})$ and left for $90 \mathrm{~min}$ at room temperature. Stock SP-B standards were diluted 1:4 in PBS-NP40 to give a working concentration of $250 \mathrm{ng} / \mathrm{mL}$. Serial dilutions using PBS-NP40 of stock SP-B and lavage samples diluted 1:20 while on ice were incubated $2 \mathrm{~h}$ along with a blank (PBS-NP40). Polyclonal rabbit antihuman SP-B antibody was diluted 1:400 in SP-B blocking buffer and incubated for $1 \mathrm{~h}$.

Purified human SP-D from human alveolar proteinosis lavage $(127 \mu \mathrm{g} / \mathrm{mL})$ was diluted in PBS to give a working concentration of $250 \mathrm{ng} / \mathrm{mL}$. Serial dilutions using PBS of standard SP-D and lavage samples diluted 1:10 were incubated overnight at $4^{\circ} \mathrm{C}$, along with a blank (PBS). SP-D blocking buffer $(1 \%$ BSA in PBS) was added to each well $(300 \mu \mathrm{L})$ and left for $1 \mathrm{~h}$. Rabbit anti-human rSP-D (neck/head), $1.0 \mathrm{mg} / \mathrm{mL}$, was diluted 1:1000 in 1\% BSA in PBS and incubated for $1 \mathrm{~h}$.

For each ELISA, goat anti-rabbit horseradish peroxidase conjugate (P0448; DAKO, Bucks, U.K.) was diluted and incubated for $1 \mathrm{~h}$. For SP-A, a 1:2000 dilution took place using $0.05 \%$ Tween $20,2.5 \%$ human albumin, and $2.5 \%$ goat serum in PBS. For SP-B, a 1:1000 dilution took place using 0.05\% Tween 20 and $1.0 \%$ human albumin in PBS. For SP-D, a 1:5000 dilution took place using 1\% BSA in PBS. After this, incubation with 3,3',5,5'-tetramethylbenzidine/peroxide took place in the dark at room temperature for $20 \mathrm{~min}$ before stopping the reaction with $2 \mathrm{~N} \mathrm{H}_{2} \mathrm{SO}_{4}$. Apart from this final stage, between each stage of every assay all wells were aspirated, washed forcefully five times with wash buffer $(0.05 \%$ Tween 20 in $10 \mathrm{mM}$ Tris, pH 8.0), and blotted dry. All reagents were added in $100-\mu \mathrm{L}$ volumes per well apart from the blocking buffer. During incubation periods, plates were covered with an adhesive strip, placed on a plate agitator, and incubated at $37^{\circ} \mathrm{C}$ unless otherwise stated. All samples and standards were analyzed in duplicate. Measurement of absorbance used wavelengths of $450 \mathrm{~nm}$ and $570 \mathrm{~nm}$, and standard curves were constructed for each assay.

Running 15 SP-A plates, 10 SP-B plates, and 11 SP-D plates, the mean (range) $r^{2}$ of logged standard curves was 0.989 (0.964-0.998), 0.991 (0.984-0.997), and 0.991 (0.979$0.996)$, respectively. The intra-assay coefficient of repeatability for 218 duplicate SP-A samples analyzed at 1:3200 dilution was $9.0 \%, 8.9 \%$ for 70 samples analyzed at 1:6400, and overall, $9.5 \%$ for 262 samples analyzed. Intra-assay coefficient of repeatability for 250 duplicate SP-B samples analyzed at $1: 20$ dilution was $12.2 \%$. Intra-assay coefficient of repeatability for 256 SP-D samples analyzed at 1:10 dilution was $11.3 \%$. Coefficient of variance for five intra-assay spikes was $4.2 \%$, $3.5 \%$, and $4.2 \%$ for SP-A, SP-B, and SP-D assays, while the interassay coefficient of variation across eight plates was $14.1 \%, 13.2 \%$, and $13.2 \%$, respectively. Interassay coefficient of variation for standard concentrations of $15.6 \mathrm{ng} / \mathrm{mL}, 62.5$ $\mathrm{ng} / \mathrm{mL}$, and $250 \mathrm{ng} / \mathrm{mL}$ was $17.0 \%, 11.9 \%$, and $10.9 \%$ for SP-A, $12.2 \%, 11.9 \%$, and $12.3 \%$ for SP-B, and $14.5 \%, 7.1 \%$, and $9.5 \%$ for SP-D, respectively.

Data analysis. Statistics were performed using SPSS 10.0 for Windows statistical package. Unless otherwise stated, numerical results are presented as median (IQR). Graphical data are plotted using box-and-whisker plots. Tests of normality of distribution took place using the one-sample KolmogorovSmirnov test. Two-tailed tests were used for all statistical comparisons. Statistical significance was defined as a $p$ value of $<0.05$.

Comparisons between groups took place using the MannWhitney $U$ test for continuous data and the $\chi^{2}$ or Fisher's exact test for categorical data. Paired data were analyzed using the Wilcoxon signed-rank test. Correlations between continuous data used Spearman's rho correlation coefficient. Assessment of duplicate measures used the method described by Bland and Altman (32). The coefficient of variation was calculated using Arcus Quickstat 1.0 statistical package. Repeated measures ANOVA was used to analyze measurements of surfactant protein concentration for the same infant over time. Natural logarithmic transformation of nonparametric data took place when necessary to allow these analyses to be carried out (resulting in normally distributed data). Data points below the level of detection of the assays were not included in the analyses.

\section{RESULTS}

Fifty infants (77\%) from the first 65 liveborn infants randomized to the surfactant trial at Liverpool Women's Hospital were enrolled to this study. Reasons for noninclusion were randomized but not requiring intubation $(n=2)$; extubation occurred before the initial sampling window $(n=5)$; routine suctioning not performed on d 1 owing to pulmonary hemorrhage or severity of condition $(n=3)$ or postnatal death $(n=$ $2)$; or operational reasons $(n=3)$. All mothers had received at least one dose of antenatal steroids, $46(92 \%)$ receiving two or more. Forty-nine (98\%) infants received a second surfactant dose after $12 \mathrm{~h}$. Twenty-five infants received poractant alfa and 25 , pumactant. Median (IQR) time after delivery to initial surfactant dose administration was $7 \mathrm{~min}$ (IQR, 5-12 min).

Table 1 shows summary demographic and outcome data for the whole cohort. Twenty (40\%) infants were born after spontaneous vaginal delivery, six (12\%) by semielective cesarean section for IUGR, and three (6\%) by assisted delivery. Of the $21(42 \%)$ infants born by emergency cesarean section, 10 
Table 1. Summary of demographic and outcome data for study infants

\begin{tabular}{lc}
\hline \multicolumn{1}{c}{$\begin{array}{c}\text { Demographic/outcome } \\
\text { variable }\end{array}$} & $\begin{array}{c}\text { Study cohort } \\
\text { unless otherwise stated })\end{array}$ \\
\hline Male (\%) & $34(68)$ \\
Gestation wk* & $27.5(1.5)$ \\
Birth weight (g)* & $914(278)$ \\
Mortality (\%) & \\
Neonatal & $9(18)$ \\
Predischarge & $15(30)$ \\
CLD at 36 wk (\%) & \\
All infants & $24(48)$ \\
Survivors at 36 wk & $24 / 40(60)$ \\
CLD or death at 36 wk (\%) & $34(68)$ \\
Among survivors to discharge & \\
Total ventilated days** & $6(3-15)$ \\
Total hospital days* & $81(56-107)$ \\
Home oxygen (\%) & $15 / 35(43)$ \\
All infants & \\
Pneumothorax (\%) & $6(12)$ \\
Pulmonary hemorrhage (\%) & $5(10)$ \\
*Mean (SD). **Median (IQR). &
\end{tabular}

$(20 \%)$ were because of pregnancy-induced hypertension or antepartum hemorrhage, six $(12 \%)$ for fetal distress, and five $(10 \%)$ because of suspected chorioamnionitis. Sixteen (32\%) of the infants were from twin deliveries. Demographic and clinical outcomes between surfactant groups in this study were similar to the overall trial findings (26).

From the whole cohort, 15 infants died before discharge. Nine were because of a primary respiratory cause of which eight had received pumactant. Fewer infants died before discharge home if they had been treated with poractant alfa (4 of 25 versus 11 of 25 ) in keeping with the overall trial findings (26). Compared with those discharged home, infants dying were lighter at birth (734 g; IQR, 600-917 g versus $920 \mathrm{~g}$; IQR, 724-1070 $\mathrm{g} ; p=0.02$ ) with higher CRIB scores (8.5; IQR, 6-13 versus 4; IQR, 2-8; $p=0.001)$. There was no significant difference in sex or gestational age between these groups. Infants alive at 36 postmenstrual weeks with CLD were born lighter (804 g; IQR, 648-99 g versus 1,005 g; IQR, $858-1,257 \mathrm{~g} ; p=0.009$ ), at lower gestational age (26 wk; IQR, 25-28 wk versus $28 \mathrm{wk}$; IQR, 27-29 wk; $p=0.008$ ), with higher CRIB scores (6; IQR, $3-8$ versus 2.5 ; IQR, $2-6$; $p$ $=0.031)$ compared with those without CLD.

Median percentage volume return of BAL fluid from all 50 infants was $50 \%$ (IQR, 42-58\%) and supernatant volume was 93\% (IQR, 91-96\%). Median SP-A concentration from all collected samples was $234 \mu \mathrm{g} / \mathrm{mL}$ (IQR, $85-463 \mu \mathrm{g} / \mathrm{mL}$ ). Figure 1 shows the change during the first 2 wk in BAL SP-A concentrations for the whole study cohort. Overall, BAL SP-A concentrations rose significantly during the first $4 \mathrm{~d}(p=0.02)$ and during the first postnatal week $(p=0.04)$. Although the median BAL SP-A concentration continued to rise in the second postnatal week, the change was not significant. Paired samples were significantly different from d 1 concentrations at all time intervals within the first $2 \mathrm{wk}$ except on $\mathrm{d} 6$.

Median SP-B concentration from all collected samples was $544 \mathrm{ng} / \mathrm{mL}$ (IQR, 147-1851 ng/mL). Figure 2 shows the change during the first $2 \mathrm{wk}$ in BAL SP-B concentrations for the whole study cohort and for the surfactant treatment groups. Overall, BAL SP-B concentrations rose significantly during the first $4 \mathrm{~d}(p<0.001)$ and during the first postnatal week $(p=$ 0.02 ). Type of surfactant significantly influenced the rise in SP-B $(p=0.001)$. Paired samples for the whole cohort were significantly different from d 1 concentrations on d 3 ( $p=$ $0.02)$, d $4(p=0.01)$, and d $6(p=0.02)$.

The median SP-D concentration from all collected samples was $872 \mathrm{ng} / \mathrm{mL}$ (IQR, 448-1894 $\mathrm{ng} / \mathrm{mL}$ ). Figure 3 shows the change during the first 2 postnatal weeks in BAL SP-D concentrations for the whole study cohort. BAL SP-D concentrations rose significantly during the first $4 \mathrm{~d}(p=0.001)$ and during the first postnatal week $(p=0.001)$. Although the

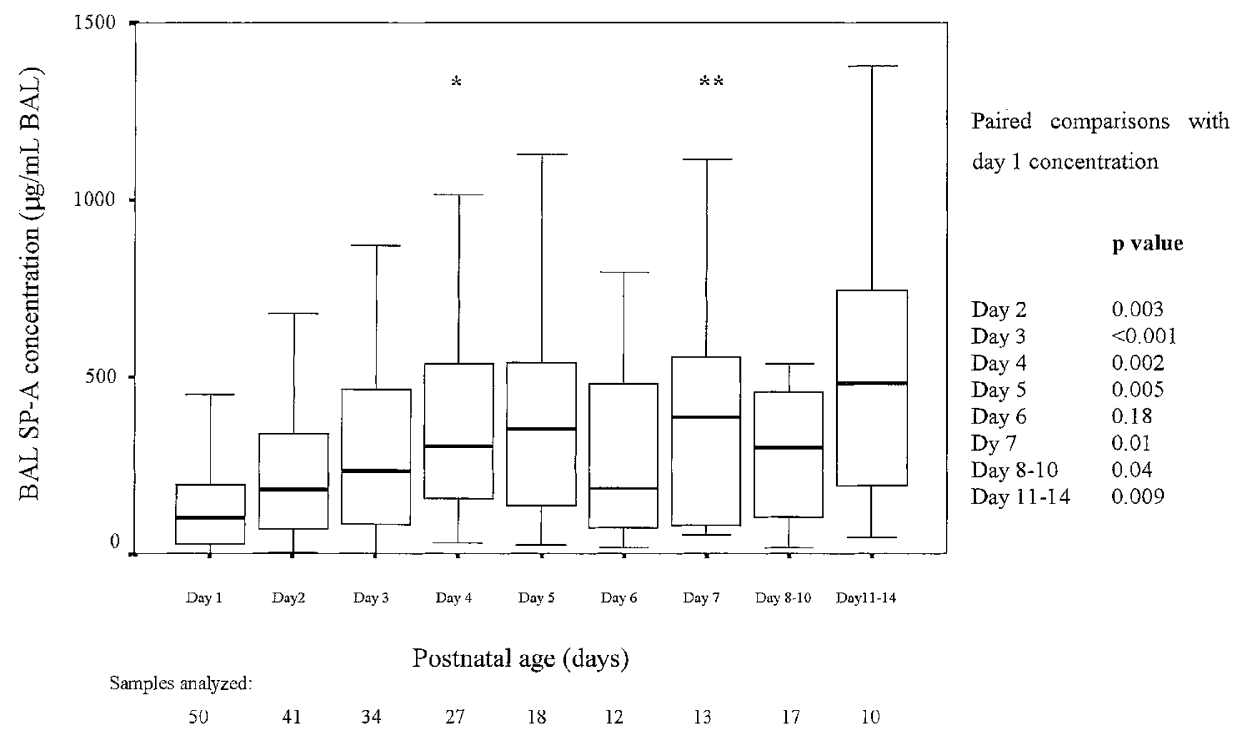

Figure 1. BAL SP-A concentration during the first $2 \mathrm{wk}$ for the whole cohort. A box-and-whisker plot of BAL SP-A concentrations for whole cohort showing significant rise in concentration during the first $4 \mathrm{~d}(* p=0.02)$ and first postnatal week $(* * p=0.04)$. Paired samples differed significantly from d 1 concentrations at all time intervals within the first 2 postnatal weeks apart from $\mathrm{d} 6$. The number of samples analyzed at each point is shown. 


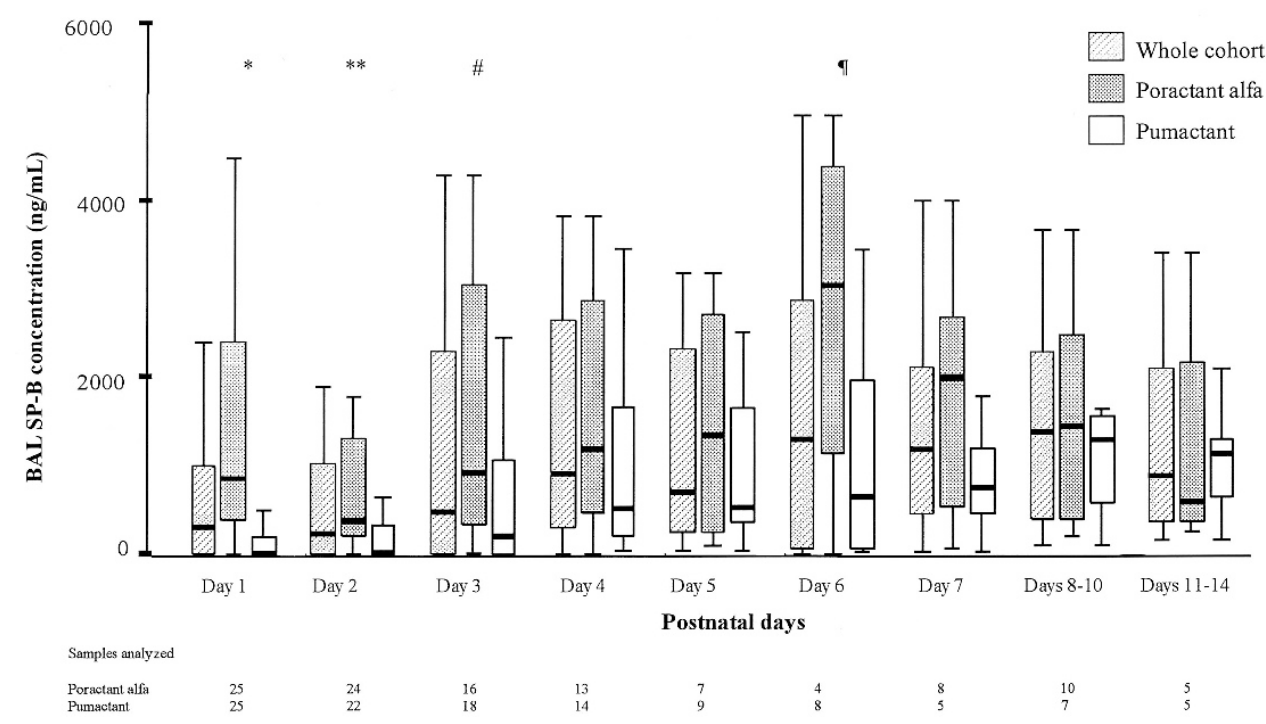

Figure 2. BAL SP-B concentration during the first 2 wk for the whole cohort and surfactant subgroups. A box-and-whisker plot of BAL SP-B concentration for the whole cohort (diagonal gray lines). Concentrations rose significantly during the first week $(\Phi p=0.02)$. In addition, box-and-whisker plots of BAL SP-B concentration for infants administered poractant alfa (hashed gray boxes) and pumactant (white boxes) are shown. BAL SP-B concentrations were significantly higher in poractant alfa- compared with pumactant-treated infants during the first 3 postnatal days $\left({ }^{*} p<0.001 ; * * p=0.04\right.$; \#p $\left.=0.02\right)$. The number of samples analyzed for each group is shown.

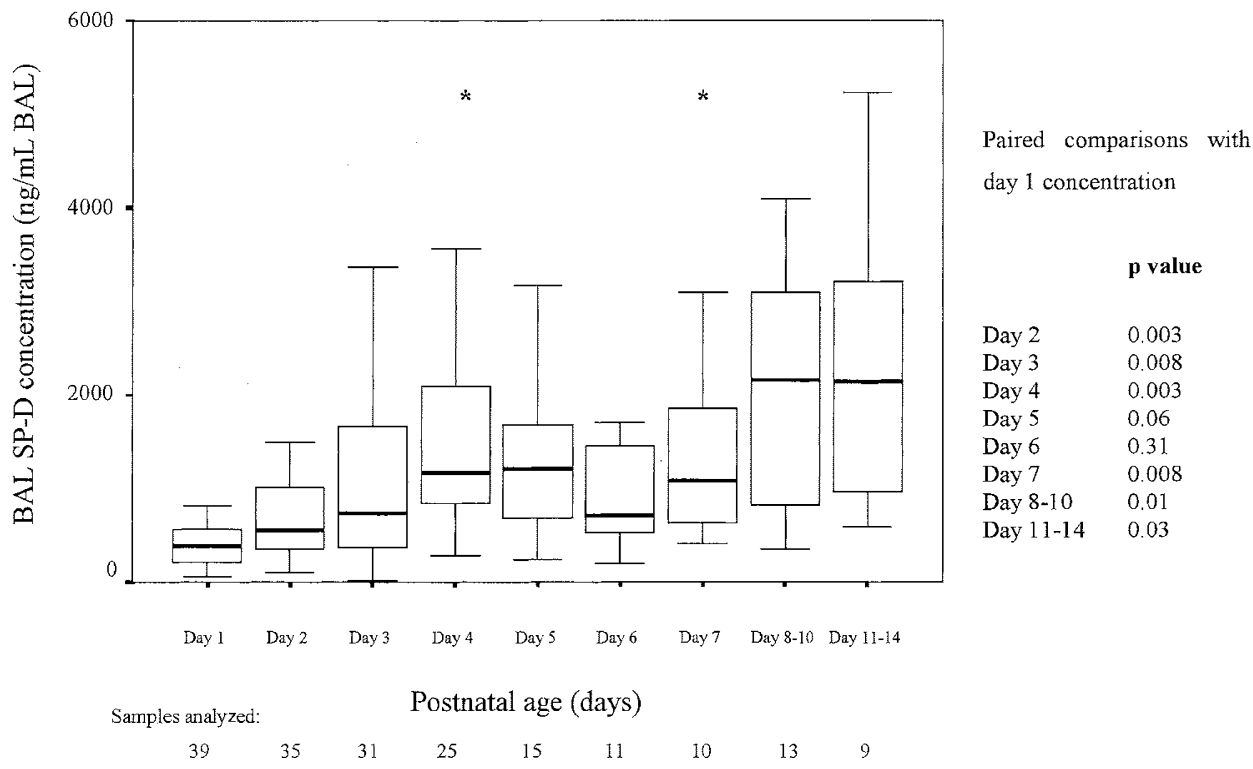

Figure 3. BAL SP-D concentration during the first $2 \mathrm{wk}$ for the whole cohort. A box-and-whisker plot of BAL SP-D concentration for whole cohort showing a significant rise in concentration during the first $4 \mathrm{~d}$ and first postnatal week $(* p=0.001)$. Paired samples differed significantly from $\mathrm{d} 1 \mathrm{concentrations}$ at most time intervals within the first 2 postnatal weeks. The number of samples analyzed at each point is shown.

median BAL SP-D concentration continued to rise until the end of the second postnatal week, the change was not significant. Paired samples were significantly different from d 1 concentrations at most time intervals within the first $2 \mathrm{wk}$.

BAL SP-A concentrations did not correlate with gestational age or birth weight at any time during the first $2 \mathrm{wk}$. There was a significant negative correlation between BAL SP-B concentration and gestational age on $\mathrm{d} 8-10\left(r^{2}=-0.525, p=\right.$ $0.031)$. BAL SP-B correlated significantly and negatively with birth weight on $\mathrm{d} 3\left(r^{2}=-0.479, p=0.004\right), \mathrm{d} 4\left(r^{2}=\right.$ $-0.398, p=0.04), \mathrm{d} 5\left(r^{2}=-0.610, p=0.01\right)$, and $\mathrm{d} 6\left(r^{2}\right.$ $=-0.742, p=0.006)$. BAL SP-D concentration correlated significantly with birth weight on $\mathrm{d} 2\left(r^{2}=0.460, p=0.005\right)$ and $\mathrm{d} 3\left(r^{2}=0.560, p=0.046\right)$ but not with gestational age. There were no significant differences in BAL SP-A or SP-D concentration between infants delivered with suspected chorioamnionitis and those without. In contrast, those with suspected chorioamnionitis had significantly higher BAL SP-B concentrations on d 2 compared with those without (2882 $\mathrm{ng} / \mathrm{mL}$; IQR, $1720-4250 \mathrm{ng} / \mathrm{mL}$ versus $181 \mathrm{ng} / \mathrm{mL}$; IQR, $5-528 \mathrm{ng} / \mathrm{mL} ; p=0.005)$.

Strong positive correlations occurred between the duration of mechanical ventilation among survivors and BAL SP-B concentration on d $5\left(r^{2}=0.892, p=0.003\right)$ and $\mathrm{d} 8-10\left(r^{2}\right.$ 
$=0.800, p=0.003)$. There were significant negative correlations between BAL SP-D concentrations and duration of ventilation among survivors on $\mathrm{d} 1\left(r^{2}=-0.388, p=0.046\right)$ and d $3\left(r^{2}=-0.605, p=0.004\right)$. There was no significant correlation among survivors between BAL SP-A concentration and the duration of ventilation at any time during the first $2 \mathrm{wk}$. BAL SP-A, SP-B, and SP-D concentrations did not differ significantly between sexes, or between infants delivered because of fetal distress or IUGR and those not delivered for these reasons.

There was no significant difference at any time in BAL SP-A or SP-D concentration between infants treated with poractant alfa and those treated with pumactant. BAL SP-B concentrations were significantly higher in poractant alfa-treated infants during the first 3 postnatal days (Fig. 2). BAL SP-B concentrations in pumactant-treated infants started very low but rose significantly during the first week $(p<0.001)$. BAL SP-B concentrations rose in the poractant alfa-treated infants during the first week but not significantly.

There was no significant difference during the first $2 \mathrm{wk}$ in BAL SP-A concentration between infants dying before discharge compared with those discharged home. In contrast, infants surviving tended to have higher BAL SP-B concentrations during the first 2 wk compared with those dying. These differences were significant on $\mathrm{d} 2(350 \mathrm{ng} / \mathrm{mL}$; IQR, 107-1250 $\mathrm{ng} / \mathrm{mL}$ versus $5 \mathrm{ng} / \mathrm{mL}$; IQR, 3-340 $\mathrm{ng} / \mathrm{mL} ; p=0.03$ ) and $\mathrm{d} 6$ (3453 ng/mL; IQR, 2280-3817 ng/mL versus $69 \mathrm{ng} / \mathrm{mL}$; IQR, $42-647 \mathrm{ng} / \mathrm{mL} ; p=0.005)$. Although BAL SP-B concentrations among infants who died started low, there was a significant rise in BAL SP-B concentration during the first $4 \mathrm{~d}(p=$ $0.01)$ and during the first postnatal week $(p=0.04)$ unlike among those infants who survived. Infants dying before discharge had generally lower BAL SP-D concentrations during the first postnatal week when compared with those being discharged home although this was statistically significant only on d $2(359 \mathrm{ng} / \mathrm{mL}$; IQR, 217-526 ng/mL versus $698 \mathrm{ng} / \mathrm{mL}$; IQR, 400-1,091 ng/mL; $p=0.04)$.

During the first 4 postnatal days there was no significant difference in BAL SP-A or SP-B concentrations between survivors developing CLD and those not. In contrast, BAL SP-D concentrations were significantly lower on $\mathrm{d} 2$ and $\mathrm{d} 3 \mathrm{among}$ survivors requiring supplementary oxygen on d 28 compared with those that did not (Fig. 4). At 36 postmenstrual weeks, a similar trend was shown, but differences were not statistically significant. Median BAL SP-A, SP-B, and SP-D concentrations continued to rise during the first 2 postnatal weeks in infants going on to develop CLD. However, group comparisons with those not developing CLD were not possible after $\mathrm{d}$ 4. Only a maximum of three survivors not developing CLD were intubated at any point after this time.

\section{DISCUSSION}

This is the first study to examine postnatal changes in BAL $\mathrm{SP}$ concentrations in preterm infants randomized to receive a natural or synthetic surfactant, using a distal NB-BAL technique (23). BAL SP-A concentrations rose significantly during the first postnatal week in these infants, in keeping with reports

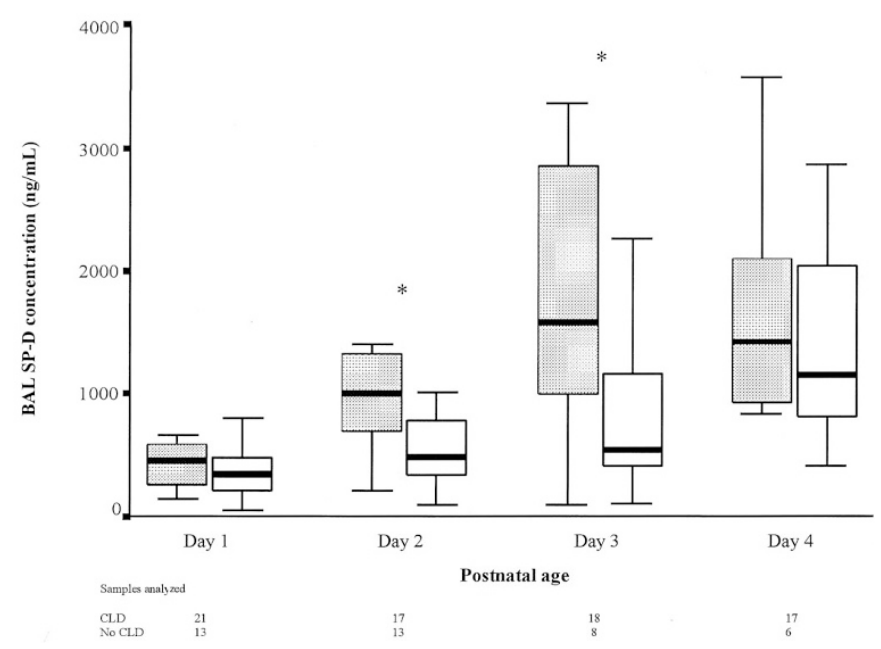

Figure 4. BAL SP-D concentration in infants with and without an oxygen requirement at 28 postnatal days. A box-and-whisker plot of BAL SP-D concentrations in infants with CLD (white boxes) and without CLD (hashed gray boxes) at 28 postnatal days. BAL SP-D concentrations were significantly lower on $\mathrm{d} 2$ and d 3 in those needing supplementary oxygen at d $28\left({ }^{*} p=0.04\right)$. The number of samples analyzed at each point for each group is shown.

using a more proximal tracheal aspirate method $(13,15,16,19$, 21). Just two reports have previously described postnatal changes in BAL SP-B concentration in $\operatorname{RDS}(14,16)$. Both used the more proximal aspirate techniques in placebocontrolled studies for a short period. Neither correlated data with outcome, and both corrected for lavage dilution using albumin as a denominator, a method generally not recommended (33). This study has measured SP-B concentrations using distal NB-BAL and a recognized method of standardizing for lavage dilution. We have shown BAL SP-B concentrations for the whole cohort rose significantly during the first postnatal week. In addition, we have demonstrated for the first time postnatal BAL SP-D concentrations in preterm infants with RDS randomized to receive surfactant. We have shown that SP-D is detectable and that concentrations rose significantly during the first postnatal week. The only previously published data describing BAL SP-D in preterm infants was in the presurfactant era (18). SP-D concentrations, of the same order of magnitude as the present study, rose postnatally although not significantly. However, dexamethasone did increase concentrations significantly. In the present study, no infant received corticosteroids in the initial postnatal period, although all mothers had received at least one dose antenatally.

This is the first study comparing BAL surfactant protein concentrations in preterm infants randomly assigned to receive natural or synthetic surfactants. There was no significant difference in BAL SP-A or SP-D concentration between poractant alfa- and pumactant-treated infants despite differences in physiologic effects $(34,35)$ and clinical outcome $(26)$. BAL SP-A concentrations do not differ between poractant alfa- and placebo-treated infants (13) or in other nonrandomized comparisons between natural and synthetic surfactants (15). Despite immunoregulatory functions of SP-A and SP-D, poractant alfa did not significantly increment their BAL concentrations when compared with a synthetic surfactant. 
As anticipated, BAL SP-B concentrations on d 1 (after surfactant therapy) were significantly higher in poractant alfatreated infants compared with pumactant-treated infants, and remained so for the first $3 \mathrm{~d}$. All but one of the poractant alfa-treated infants $(96 \%)$ received exactly two doses, administered on a dose per kilogram basis at birth and $12 \mathrm{~h}$. Quantification of BAL SP concentration before surfactant administration was not possible owing to the unit's policy of giving surfactant therapy in the delivery suite as soon after delivery as possible. In non-SP-B-supplemented airways (pumactant treated), d 1 median BAL SP-B concentrations were lower by almost a factor of 100 compared with infants receiving a surfactant containing SP-B. Measured BAL SP-B concentration in poractant alfa-treated infants did not rise significantly during the first postnatal week. This may be caused by masking of postnatal increases in endogenous production of SP-B by exogenous supplementation of SP-B. In contrast, BAL SP-B concentrations rose significantly during the first postnatal week in pumactant-treated infants. Although after d 3 BAL SP-B concentration did not differ significantly between treatment groups, median SP-B concentrations in pumactant-treated infants only reached those of poractant alfa treatment in the second postnatal week. Therefore, although postnatal increase in BAL SP-B takes place in the absence of SP-B augmentation, there is relative deficiency of SP-B in pumactant-treated infants in the initial postnatal period and this is associated with worse clinical outcome in this group (26). The poor prognosis of congenital SP-B deficiency underlines the importance of SP-B to clinical outcome (36). Infants surviving had higher median BAL SP-B concentrations during the first postnatal week compared with those dying. However, infants dying had a significant increase in BAL SP-B during the first $7 \mathrm{~d}$ in contrast to those surviving. Outcome was therefore inversely associated with concentration of BAL SP-B rather than increment in concentration alone. There was no significant difference in BAL SP-B concentration between those developing, and those not developing CLD, although short duration of ventilation among survivors and high mortality reduced the comparative data available. In addition, the study was not powered to detect significant differences in BAL SP-B concentrations for CLD. There was marked variation in BAL SP-B concentration between and within infants developing CLD, reflecting differences between surfactant therapies and similar incidences of CLD between treatment groups.

Despite data indicating an important contributory role of SP-A to surfactant metabolism and function, there was no demonstrable difference in BAL SP-A concentration related to clinical outcomes such as death or development of CLD. This may be related to a number of reasons. The action of SP-A, including its synergistic role with SP-B, occurs even at very low concentrations. The specific characteristic of SP-A in counteracting surfactant inhibition by plasma proteins may be undemonstrable in this high-risk group of infants with universally leaky airways. High mortality rate and short duration of ventilation among survivors without CLD reduced the available comparative data between clinical outcome groups. The multiplicity of factors contributing to outcome measures such as death and CLD in this very preterm population may have masked the relative contribution SP-A deficiency might have made. Also, sample size of this observational study was not powered to detect significant differences in BAL SP-A concentrations between outcome groups. However, from these data, additional supplementation of SP-A to exogenous surfactant seems unlikely to have a significant effect on these outcomes. Indeed, respiratory function in SP-A knockout mice appears normal despite having defects in innate lung immunity and surfactant more easily inhibited by plasma proteins (37).

BAL SP-D concentrations were lower among infants dying, significantly so on d 2, when compared with survivors. Furthermore, concentrations were lower in infants who developed CLD by 28 postnatal days compared with those who did not. There were significant early negative correlations between BAL SP-D concentration and subsequent duration of ventilation among survivors. The association between worse clinical outcome and lower SP-D concentrations may relate to the contribution SP-D makes to innate defense by altering cytokine production, enhancing leukocyte chemotaxis and function, and regulating lymphocyte production and apoptosis $(2,9)$. Alternatively, data from SP-D knockout mice indicate a role for SP-D in surfactant homeostasis (10). Further exploration of the contribution SP-D makes to surfactant function is needed.

The methodology of BAL has important limitations that must be considered when interpreting data findings. There is no universally accepted method to standardize for dilution of alveolar proteins during BAL (23). In the absence of such a marker consensus documents recommend results be expressed as concentration per milliliter of BAL fluid (33). Percentage BAL fluid return was comparable to recommended values (23, $33)$. The technique and site of sampling will alter measured concentrations. A distal sampling method of BAL was adopted following accepted methodology to facilitate investigation of the alveolar disease processes (23). As might be expected, BAL SP-A concentrations on d 1 in this study were higher when compared with concentrations reported from studies using a more proximal tracheal aspiration technique but the same denominator of lavage dilution $(13,18)$. However, concentrations were of the same order of magnitude between studies (micrograms per milliliter of BAL fluid). As with other published data in this field, considerable variation within and between infants was noted (13-15). Problems associated with recovery, processing, and quantification of lavage constituents in this, as in all studies of preterm ventilated infants, will contribute to this variation. Other methods of investigation (e.g. immunohistochemistry) may be performed simultaneously to substantiate the findings, but were beyond the scope of this present study.

For ethical reasons, an inherent limitation of using BAL as an investigative technique is obtaining samples from preterm infants recovering from RDS. The number of infants remaining intubated on whom BAL could be performed decreased with each successive postnatal day (see Figs. 1-4), in part because of mortality, but also because of recovery and extubation. Few survivors not developing CLD remained intubated after $\mathrm{d} 4$. The effect of prematurity on lung defense mechanisms remains poorly understood. To contrast potential differences between disease states, only infants born at $<30$ wk gestation were 
studied to ensure gestational age-matched controls. However, statistical comparisons of BAL SP concentrations with demographic and clinical variables will also reflect the available sample size at that point. For this reason, subanalyses based on gestational age or birth weight were not performed. The overall study sample size could have limited the power of the study to detect significant differences in BAL SP concentrations between surfactant and outcome groups. There were no published NB-BAL data available [using an appropriate denominator of BAL dilution (33)] from this very preterm population treated with poractant alfa and pumactant before commencing this study. Formal sample size calculation was therefore not possible. A sample size of 50 was chosen pragmatically and prospectively to be among the largest study in this field.

\section{CONCLUSIONS}

In conclusion, this study has demonstrated for the first time postnatal BAL SP concentrations in preterm infants with RDS randomized to receive a natural or synthetic surfactant using distal NB-BAL methodology. SP-D was detectable, and along with SP-A and SP-B, BAL concentrations rose significantly during the first postnatal week. There was no significant difference in BAL SP-A and SP-D concentrations between surfactants. BAL SP-A concentrations did not relate significantly with outcome, but lower BAL SP-B and SP-D concentrations were associated with worse clinical prognosis.

\section{REFERENCES}

1. Creuwels LAJM, van Golde LMG, Haagsman HP 1997 The pulmonary surfactant system: biochemical and clinical aspects. Lung 175:1-39

2. Davies J, Turner M, Klein N 2001 The role of the collectin system in pulmonary defence. Paediatr Respir Rev 2:70-75

3. King RJ, Klass DJ, Gikas EG, Clements JA 1973 Isolation of apoproteins from canine surface active material. Am J Physiol 224:788-795

4. Floros J, Kala P 1998 Surfactant proteins: molecular genetics of neonatal pulmonary diseases. Annu Rev Physiol 60:365-384

5. Yamada T, Ikegami M, Tabor BL, Jobe AH 1990 Effects of surfactant protein A on surfactant function in preterm ventilated rabbits. Am Rev Respir Dis 142:754-757

6. Kuroki Y, Voelker DR 1994 Pulmonary surfactant proteins. J Biol Chem 269:2594325946

7. Hallman M, Merritt TA, Akino T, Bry K 1991 Surfactant protein A, phosphatidylcholine, and surfactant Inhibitors in epithelial lining fluid. Am Rev Respir Dis 144:1376-1384

8. Seeger W, Grube C, Gunther A, Schmidt R 1993 Surfactant inhibition by plasma proteins: differential sensitivity of various surfactant preparations. Eur Respir J 6:971-977

9. Vaandrager AB, van Golde LMG 2000 Lung surfactant proteins A and D in innate immune defence. Biol Neonate 77(suppl):9-13

10. Ikegami M, Whitsett JA, Jobe A, Ross G, Fisher J, Korfhaegn T 2000 Surfactant metabolism in SP-D gene-targeted mice. Am J Physiol Lung Cell Mol Physiol 279:L468-L476

11. Kala P, Have TT, Nielsen H, Dunn M, Floros J 1998 Association of pulmonary surfactant protein A (SP-A) gene and respiratory distress syndrome: interaction with SP-B. Pediatr Res 43:169-177

12. Hamvas A, Nogee LM, deMello DE, Cole FS 1995 Pathophysiology and treatment of surfactant protein-B deficiency. Biol Neonate 67(suppl 1):18-31
13. Stevens PA, Schadow B, Bartholain S, Segerer H, Obladen M 1992 Surfactant protein $\mathrm{A}$ in the course of respiratory distress syndrome. Eur J Pediatr 151:596-600

14. Chida S, Phelps DS, Cordle C, Soll R, Floros J, Taeusch HW 1988 Surfactantassociated proteins in tracheal aspirates of infants with respiratory distress syndrome after surfactant therapy. Am Rev Respir Dis 137:943-947

15. Moya FR, Montes HF, Thomas VL, Mouzinho AM, Smith JF, Rosenfeld CR 1994 Surfactant protein A and saturated phosphatidylcholine in respiratory distress syndrome. Am J Respir Crit Care Med 150:1672-1677

16. Gerdes J, Whitsett J, Long W 1992 Elastase activity and surfactant protein concentration in tracheal aspirates from neonates receiving synthetic surfactant. J Pediatr 120:S34-S39

17. Kari MA, Akino T, Hallman M 1995 Prenatal dexamethasone and exogenous surfactant therapy: surface activity and surfactant components in airway specimens. Pediatr Res 38:676-684

18. Wang J-Y, Yeh T-F, Lin Y-C, Miyamura K, Holmskov U, Reid KBM 1996 Measurement of pulmonary status and surfactant protein levels during dexamethasone treatment of neonatal respiratory distress syndrome. Thorax 51:907-913

19. Gerdes JS, Abbasi S, Karp K, Hull W, Whitsett JA 1990 Surfactant protein-A in bronchoalveolar lavage fluid from neonates with RDS on conventional and highfrequency oscillatory ventilation. Pediatr Pulmonol 9:166-169

20. Lotze A, Whitsett JA, Kammerman LA, Ritter M, Taylor GA, Short BL 1990 Surfactant protein A concentrations in tracheal aspirate fluid from infants requiring extracorporeal membrane oxygenation. J Pediatr 116:435-440

21. Corcoran JD, Sheehan O, O'Hare MMT, Halliday HL 1995 Tracheal surfactant protein A following treatment with Curosurf. Appl Cardiopulm Pathophysiol 5:245248

22. Rennard SI, Basset G, Lecoossier D, O’Donnell KM, Pinkston P, Martin PG, Crystal RG 1986 Estimation of volume of epithelial lining fluid recovered by lavage using urea as marker of dilution. J Appl Physiol 60:532-538

23. Kotecha S 1999 Bronchoalveolar lavage of newborn infants. Pediatr Pulmonol Suppl 18:122-124

24. Egberts J, Brand R, Walti H, Bevilacqua C, Bréart G, Gardini F 1997 Mortality, severe respiratory distress syndrome and chronic lung disease of the newborn are reduced more after prophylactic than after therapeutic administration of the surfactant Curosurf. Pediatrics 100:e4

25. [No authors listed] 1987 Ten centre trial of artificial surfactant (artificial lung expanding compound) in very premature babies. Ten Centre Study Group. BMJ 294:991-996

26. Ainsworth SB, Beresford MW, Milligan DWA, Shaw NJ, Matthews JNS, Fenton AC, Ward Platt MP 2000 Pumactant and poractant alfa for treatment of respiratory distress syndrome in neonates born at 25-29 weeks' gestation: a randomised trial. Lancet 355:1387-1392

27. Awasthi S, Coalson JJ, Crouch E, Yang F, King RJ 1999 Surfactant proteins A and $\mathrm{D}$ in premature baboons with chronic lung injury (bronchopulmonary dysplasia): evidence for an inhibition of secretion. Am J Respir Crit Care Med 160:942-949

28. Levene MI 1981 Measurement of the growth of the lateral ventricles in preterm infants with real-time ultrasound. Arch Dis Child 56:900-904

29. Papile LA, Burstein J, Burstein R, Koffler H 1978 Incidence and evolution of subependymal and intraventricular hemorrhage: a study of infants of birth weight less than 1500 g. J Pediatr 92:529-534

30. Kerr M, Paton JY 1999 Surfactant protein levels in severe respiratory syncytial virus infection. Am J Respir Crit Care Med 159:1115-1118

31. Wright SM, Hockey PM, Enhorning G, Strong P, Reid KB, Holgate ST, Djukanovic R, Postle AD 2000 Altered airway surfactant phospholipid composition and reduced lung function in asthma. J Appl Physiol 89:1283-1292

32. Bland JM, Altman DG 1986 Statistical methods for assessing agreement between two methods of clinical measurement. Lancet 1:307-310

33. de Blic J, Midulla F, Barbato A, Clement A, Dab I, Eber E, Green C, Grigg J, Kotecha S, Kurland G, Pohunek P, Ratjen F, Rossi G 2000 Bronchoalveolar lavage in children. ERS Task Force on bronchoalveolar lavage in children. European Respiratory Society. Eur Respir J 15:217-231

34. Takahashi A, Nemoto T, Fujiwara T 1994 Biophysical properties of protein-free, totally synthetic pulmonary surfactants, ALEC and Exosurf, in comparison with surfactant TA. Acta Paediatr Jpn 36:613-618

35. Corcoran JD, Berggren P, Sun B, Halliday HL, Robertson B, Curstedt T 1994 Comparison of surface properties and physiological effects of a synthetic and a natural surfactant in preterm rabbits. Arch Dis Child Fetal Neonatal Ed 71:F165-F169

36. Chetcuti PJ, Ball RJ 1995 Surfactant apoprotein B deficiency. Arch Dis Child Fetal Neonatal Ed 73:F125-F127

37. Ikegami M, Korfhagen TR, Whitsett JA, Bruno MD, Wert SE, Wada K, Jobe AH 1998 Characteristics of surfactant from SP-A-deficient mice. Am J Physio 275:L247-L254 